\title{
I nhalt.
}

I. Der von Kürenberc . . . . . . . . . . . . . . . 1

II. Hêr Dietmâr von Aiste . . . . . . . . . . . . . 3

III. Spervogel I (Anonymus, Herigêr) . . . . . . . . . . 5

IV. Hêr Meinlôh von Sevelingen . . . . . . . . . . . . 10

V. Der burcgrâve von Regensburc . . . . . . . . . . . 11

VI. Der burcgrâve von Rietenburc . . . . . . . . . . . 12

VII. Hêr Heinrich von Veldeke . . . . . . . . . . . . . 12

VIII. Hêr Fridrich von Hasen . . . . . . . . . . . . . 17

IX. Grâve Ruodolf von Fenis . . . . . . . . . . . . . . . 22

X. Hêr Heinrich von Rugge . . . . . . . . . . . . . 23

XI. Hêr Albreht voh Jôhansdorf . . . . . . . . . . . . 28

XII. Hêr Bernger von Horheim . . . . . . . . . . . . . 30

XIII. Der von Kolmas . . . . . . . . . . . . . . . . . . 32

XIV. Hêr Heinrich von Môrungen . . . . . . . . . . . . 33

XV. Hêr Reinmâr . . . . . . . . . . . . . . . . . . 43

XVI. Spervogel II . . . . . . . . . . . . . . . . . . . . . . 59

XVII. Hêr Blîggêr von Steinach . . . . . . . . . . . . . 61

XVIIl. Hêr Hartman von Ouwe . . . . . . . . . . . . . 62

XIX. Der Marcgrâve von Hôhenburc . . . . . . . . . . . 67

XX. Hêr Hiltbolt von Swanegou . . . . . . . . . . . . 68

XXI. Hêr Walther von der Vogelweide . . . . . . . . . . 72

XXII. Hêr Wolfram von Eschenbach . . . . . . . . . . . 98 
XXIII Hêr Heinrich von Frỡenberc 101

XXIV. Der tugenthafte Schrîber . . . . . . . . . . . 102

XXV. Hêr Nithart . . . . . . . . . . . . . . . . 103

XXVI. Grâve Otte von Botenlouben . . . . . . . . . . 123

XXVII. Der herzoge von Anehalt . . . . . . . . . . . 125

XXVIII. Hêr Liutolt von Savene . . . . . . . . . . . . 126

XXIX. Hêr Reimâr der Videler . . . . . . . . . . . . . . . . 129

XXX. Der Truhsæze von Sant Gallen . . . . . . . . . . . 129

XXXI. Grâve Friderich von Lîningen . . . . . . . . . 135

XXXII. Hêr Kristân von Hamle . . . . . . . . . . . . 136

XXXIII. Hêr Uolrich von Liehtenstein . . . . . . . . . . 139

XXXIV. Hêr Burkart von Hôhenvelṣ . . . . . . . . . . . 148

XXXV. Der burcgrâve von Lüenz . . . . . . . . . . . 153

XXXVI. Hêr Gotfrit von Nifen . . . . . . . . . . . . 155

XXXVII. Der Taler . . . . . . . . . . . . . . . . . 161

XXXVIII. Schenk Uolrich von Wintersteten . . . . . . . . 161

XXXIX. Der von Sahsendorf . . . . . . . . . . . . . 172

XL. Hêr Reinmâr von Zweter . . . . . . . . . . . 173

XLI. Bruoder Wernher . . . . . . . . . . . . . . 178

XLII. Der Marner . . . . . . . . . . . . . . . . . . . . 179

XLIII. Hêr Ruodolf von Rôtenburc . . . . . . . . . . . 183

XLIV. Der Schenke von Limpurc . . . . . . . . . . . 188

XLV. Der Hardegger . . . . . . . . . . . . . . . . . 190

XLVI. Hêr Reinmâr von Brennenbere . . . . . . . . . 190

XLVII. Der Tanhôser . . . . . . . . . . . . . . . . 193

XLVIII. Grâve Kraft von Toggenburc . . . . . . . . . . 199

XLIX. Hèr Hûc von Werbenwâc . . . . . . . . . . . 200

L. Hêr Walther von Metze . . . . . . . . . . . . 202

LI. Hêr Rubin . . . . . . . . . . . . . . . . . 203

LII. Hêr Wahsmuot von Mälnhûsen . . . . . . . . . 205

LIII. Marcgrâve Heinrich von Mîssen . . . . . . . . . 206

LIV. Der von Schsrpfenberc . . . . . . . . . . . . 207

LV. Hêr Wahsmuot von Kunzich . . . . . . . . . . 208

LVI. Gedrât . . . . . . . . . . . . . . . . . . 209

LVII. Hêr Geltâr . . . . . . . . . . . . . . . . . 210

LVIII. Der von Wildonje . . . . . . . . . . . . . . 211 
LIX Der von Snonegge sulto

LX. Meister Heinrich Teschler . . . . . . . . . . 212

LXI. Hêr Heinrich von Stretelingen . . . . . . . . . 213

LXII. Meister Friderich von Sunburc . . . . . . . . . 214

LXIII. Meister Sigehêr . . . . . . . . . . . . . . 216

LXIV. Hêr Walther von Klingen . . . . . . . . . . 218

LXV. Künic Kuonrât der junge . . . . . . . . . . . 220

LXVI. Meister Rûmzlant . . . . . . . . . . . . . . 220

LXVII. Meister Singûf . . . . . . . . . . . . . . . 221

LXVIII. Meister Stolle . . . . . . . . . . . . . . . 223

LXIX. Meister Kuonrât von Würzebure . . . . . . . . 223

LXX. Boppe . . . . . . . . . . . . . . . . . . 226

LXXI. Der wilde Alexander . . . . . . . . . . . . 227

LXXII. Hêr Kuonrât der Schenke von Landegge . . . . . 232

LXXIII. Der Schnolmeister von Ezzelingen . . . . . . . 236

LXXIV. Süezkint der jude von Trimberc . . . . . . . . 237

LXXV. Der von Trôstberc . . . . . . . . . . . . . 238

LXXVI. Hêr Steimâr . . . . . . . . . . . . . . . . 239

LXXVII. Der Kanzeler . . . . . . . . . . . . . . . 243

LXXVIII. Herman der Damen . . . . . . . . . . . . . 245

LXXIX. Meister Heinrich Vrouwenlop . . . . . . . . . 247

LXXX. Marcgrâve Otte von Brandenburc mit dem pfile . . 257

LXXXI. Herzoge Heinrich von Pressela . . . . . . . . 257

LXXXII. Herzoge Jôhans von Brabant . . . . . . . . . 259

LXXXIII. Kunic Wenzel von Beheim . . . . . . . . . . 261

LXXXIV. Wizlâv . . . . . . . . . . . . . . . . . . 262

LXXXV. Grâve Kuonrât von Kilchberc . . . . . . . . . 265

LXXXVI. Grâve Wernher von Hônberc . . . . . . . . . 267

LXXXVII. Meister Jôhans Hadloub . . . . . . . . . . . 268

LXXXVIII. Der von Bûwenbure . . . . . . . . . . . . . 277

LXXXIX. Der Guotære . . . . . . . . . . . . . . . 278

XC. Der Dürner . . . . . . . . . . . . . . . . 279

XCI. Hêr Kuonrât von Altsteten . . . . . . . . . . 280

XCII. Hêr Kristân von Lupîn . . . . . . . . . . . 281

XCIII. Hêr Heinrich Hetzbolt von Wîzensê . . . . . . . 282

XCIV. Regenboge . . . . . . . . . . . . . . . . 283 
XCV. Albreht marchschal von Raprehtswîle $\quad 284$

XCVI. Hêr Otte zem Turne . . . . . . . . . . . . . . 285

XCVII. Heinrich von Mugelîn . . . . . . . . . . . . . . 286

XCVIII. Namenlose Lieder . . . . . . . . . . . . . . . 288

Anmerkangen . . . . . . . . . . . . . . . . . . . . 310

Glossar . . . . . . . . . . . . . . . . . . . . . . . 381

Namenverzeichnis . . . . . . . . . . . . . . . . . . . 402 